\title{
Unmanned Aerial Vehicles in Interhospital Transport of Critically Ill Cardiovascular Patients under Extreme Weather Conditions
}

\author{
You Zhang; ${ }^{1}$ Wei Yang; ${ }^{1}$ Hongchao Kang; ${ }^{2}$ Qiaofang Yang; ${ }^{2}$ Chuanyu Gao ${ }^{1} \odot$
}

1. Department of Cardiology, Fuwai Central China Cardiovascular Hospital, Central China Fuwai Hospital of Zhengzhou University, Henan Provincial People's Hospital Heart Center, Henan Key Laboratory for Prevention and Control of Coronary Heart Disease, Central China Branch of National Center for Cardiovascular Diseases, Zhengzhou, Henan Province, China

2. Fuwai Central China Cardiovascular Hospital, Central China Fuwai Hospital of Zhengzhou University, Henan Provincial People's Hospital Heart Center, Zhengzhou, Henan Province, China

\author{
Correspondence: \\ Chuanyu Gao \\ Department of Cardiology \\ Fuwai Central China Cardiovascular Hospital \\ Central China Fuwai Hospital of \\ Zhengzhou University \\ Henan Provincial People's Hospital \\ Heart Center \\ No. 1 Fuwai Road, Zhengzhou, \\ Henan 451464, China \\ E-mail: gaocy6802@zzu.edu.cn
}

Conflicts of interest: The authors declare none.

Keywords: cardiovascular disease; critical care; extreme weather; patient transfer; unmanned aerial vehicles

\section{Abbreviations: \\ Hospital F: Fuwai Central China Cardiovascular Hospital \\ Hospital H: Henan Provincial People's Hospital ICU: intensive care unit \\ UAV: unmanned aerial vehicle}

Received: November 19, 2021

Accepted: December 5, 2021

Zhang Y, Yang W, Kang H, Yang Q, Gao C. Unmanned aerial vehicles in interhospital transport of critically ill cardiovascular patients under extreme weather conditions. Prehosp Disaster Med. 2022;37(2):294-296.

With the application and development of intelligence and information technology, unmanned aerial vehicle (UAV) technology has made remarkable research achievements and has been applied in many industries. However, there are few reports on UAVs in patient transfer under extreme weather conditions. ${ }^{1}$ This letter is aimed to share the experience with UAVs as relay communications to help transport critically ill cardiovascular patients trapped in floods.

Hospital F (Fuwai Central China Cardiovascular Hospital; Zhengzhou, Henan Province, China) is a 1,000-bed tertiary teaching hospital newly built in 2017, and also the heart center of Hospital H (Henan Provincial People's Hospital; Zhengzhou, Henan Province, China), which is a tertiary general hospital in central China's Henan Province founded in 1904. The helicopter transfer service began in 2016. The two hospitals are 25.6 kilometers apart, normally taking eight minutes by helicopter or 30 minutes by ground ambulance.

On July 20, 2021, Zhengzhou, capital of Henan Province, was lashed by torrential rain with a 24 -hour rainfall of $457.5 \mathrm{~mm}$, breaking the historical extreme value. The worst-hit Hospital $\mathrm{F}$ was inundated by floods around 3:00AM on July $21 .^{2}$ Power, water, and communications were all disrupted. Three intensive care units (ICUs) were then powered by diesel generators. At that time, there were 65 critically ill cardiovascular patients in the hospital (Table 1). Hospital F quickly decided to evacuate them to Hospital $\mathrm{H}$.

However, the inability to communicate with Hospital $\mathrm{H}$ seriously affected patient transfer. With the urgent request from the Ministry of Emergency Management, a Wing Loong $2 \mathrm{H}$ disaster-assistance drone was sent to provide communication links to Hospital $\mathrm{F}$ from Chengdu. The drone activated a mini signal transmission device to allow people inside the hospital to connect to the China Mobile network, enabling them to report their situations, call for help, or coordinate evacuation work. ${ }^{3}$

According to the risk assessment undertaken by senior physicians, 34 critically ill patients were quickly transported by helicopter and the others by boat and ambulance (they were first transferred by inflatable boat to the land approximately five kilometers away, and then were transported by ground ambulance to Hospital H). Two helicopters were involved in the transfer, equipped with a portable emergency ventilator with additional supply of oxygen and air, routine monitoring devices, and drugs. Noise cancelling headphones were also provided. Patients with extracorporeal membrane oxygenation and/or intra-aortic balloon

\section{doi:10.1017/S1049023X22000267}

(C) The Author(s), 2022. Published by Cambridge University Press on behalf of the World Association for Disaster and Emergency Medicine. This is an Open Access article, distributed under the terms of the Creative Commons AttributionNonCommercial-NoDerivatives licence (https:// creativecommons.org/licenses/by-nc-nd/4.0/), which permits non-commercial re-use, distribution, and reproduction in any medium, provided the original work is unaltered and is properly cited. The written permission of Cambridge University Press must be obtained for commercial re-use or in order to create a derivative work. 




Table 1. Patient Characteristics and 30-Day Post-Transport Outcome Note: Data are presented as number (\%) or median (interquartile range).

Abbreviations: ECMO, extracorporeal membrane oxygenation; IABP, intra-aortic balloon pump; ICU, intensive care unit.

pump were transported with the devices. This transport was directed by two trained leaders from each hospital. Every patient was escorted by two trained medical staff during transport and remained under the care of the same physician post-transport. The transfer began at 2:00PM on July 21 and finished at 7:30PM on July 22. All transports were free to patients.

Life-threatening events (eg, cardiac arrest, refractory hypoxemia or hypotension, and accidental extubation) were recorded. No deaths occurred during transport, but refractory hypotension occurred in two ST-segment elevation myocardial infarction patients complicated with cardiogenic shock during transport. One died and the other abandoned treatment because of terminal status within 24 hours of admission. Within 30 days after admission, five more patients abandoned treatment in terminal status and ICU mortality of the study population was $10.8 \%$. A total of 59 (90.8\%) patients were discharged (Table 1).

This combined transport is the first large-scale, interhospital emergency transport of critically ill cardiovascular patients in China, challenged by multiple issues: no phone signal, no power, no elevator, limited diesel, and bad weather. Luckily, all patients were safely transferred out. The UAV played an extremely important role in the patient transfer. First, it has the capability to respond quickly to disasters and strong adaptability to the environment and meteorological conditions. In this rescue, the drone accelerated the transfer and easily solved the problem of flood preventing emergency communications support vehicles from reaching hospitals. Second, it can realize the communication relay function, quickly recover the local communication on site, which then ensured the smooth operation of patient transport. ${ }^{4}$ Third, it can collect the field data, quickly transmit the visual and audio information to the command center, making judgment and decision making more timely and more correct, making more rapid response, and all departments and all kinds of rescue personnel coordination will be more adequate. Two helicopters, more than 450 inflatable boats, more than 100 rescue vehicles, and more than 3,000 volunteers were involved in this patient transfer, which was completed before dark. Finally, it made the patients trapped in the floods able to timely get in touch with their family, alleviating psychological panic and enabling them to cooperate with the transfer. This report reflects the capability of UAVs to respond to disaster situations in China. 


\section{References}

1. Carrillo-Larco RM, Moscoso-Porras M, Taype-Rondan A, Ruiz-Alejos A, BernabeOrtiz A. The use of unmanned aerial vehicles for health purposes: a systematic review of experimental studies. Glob Health Epidemiol Genom. 2018;3:e13.

2. Rescuers transfer patients from flooded hospital in Henan. China Daily Web site. https://www.chinadaily.com.cn/a/202107/22/WS60f97bfaa310efa1bd663ca7.html. Accessed November 19, 2021.
3. Drones restore telephone services. China Daily Web site. https://www.chinadaily.com. cn/a/202107/23/WS60f92bfda310efa1bd663c07.html. Accessed November 19, 2021.

4. Luman, J, Luman, B. Exploring the utilization of small unmanned aerial vehicles known as drones in early phase disaster response. Prehosp Disaster Med. 2019;34(S1): S132. 